\title{
How Does Naked Singularity Look?
}

\author{
Ken-ichi Nakao*, Naoki Kobayashi and Hideki Ishihara ${ }^{\dagger}$ \\ Department of Physics, Graduate School of Science, Osaka City University \\ Osaka 558-8585, Japan
}

\begin{abstract}
There are non-radial null geodesics emanating from the shell focusing singularity formed at the symmetric center in a spherically symmetric dust collapse. In this article, assuming the self-similarity in the region filled with the dust fluid, we study these singular null geodesics in detail. We see the time evolution of the angular diameter of the central naked singularity and show that it might be bounded above by the value corresponding to the circular null geodesic in the Schwarzschild spacetime. We also investigate the angular frequency of a physical field which propagates along the singular null geodesic and find that it depends on the impact parameter. Further, we comment on the non-uniformity of the topology of the central naked singularity.

PACS number(s): 04.20.Dw, 04.20.Gz
\end{abstract}

\footnotetext{
*e-mail: knakao@sci.osaka-cu.ac.jp

†e-mail: ishihara@sci.osaka-cu.ac.jp
} 


\section{INTRODUCTION}

Gravitational collapse is one of the most important issues in general relativity and in the theoretical research for this phenomenon, the Lemaître-Tolman-Bondi(LTB) solution which describes the motion of spherically symmetric dust fluid has played an important role. This is a very simple analytic solution of Einstein equations but has substantial physical contents. The simplest but physically significant member of this solution is the case of a homogeneous dust sphere, which is called the Oppenheimer-Snyder solution. This solution is the first example to give an elegant picture of a black-hole formation and led to the so-called cosmic censorship conjecture; roughly speaking, this conjecture states that spacetime singularities except for the Big Bang are not visible for any observer if it is resulted from physically reasonable initial conditions [1]. However, this conjecture has not yet been proven although it plays very crucial roles in the proof of the singularity theorems [2-4] and the area theorem

of black hole [5]. Rather LTB solution seems to be a candidate of the counterexample of this conjecture. Eardley and Smarr pointed out that a shell focusing singularity formed at the symmetric center in an inhomogeneous dust sphere can be naked, i.e., visible in principle [6]. After this discovery, several theoretical efforts revealed the genericity of the shell focusing naked singularity formation in the LTB solution [7-12]; there are radial null geodesics emanating from the central shell focusing singularity.

The central naked singularity of the dust sphere seems to be a "point" since it forms from an infinitesimal portion at the symmetric center of the dust sphere. However, it is not true. Recently, Mena and Nolan showed that there are non-radial null geodesics emanating from this central naked singularity [13], where non-radial means that it has a nonvanishing angular momentum. Deshingkar, Joshi and Dwivedi (DJD) studied numerically the trajectories of those geodesics within the dust fluid in detail [14]. More recently, Nolan and Mena studied various invariant quantities associated with this central naked singularity and further discussed the topology of this naked singularity [15].

It is the main purpose of this article to reveal how the central naked singularity is observed 
for distant observers outside the dust sphere. For this purpose, we investigate both the radial and non-radial null geodesics emanating from the central naked singularity in the self-similar dust sphere. Assuming that the dust sphere has a finite mass, we investigate the angular diameter of the naked singularity, or equivalently, the largest impact parameter of the null geodesics from the naked singularity; we show its time evolution. We also clarify the nonuniformity of topology of the central naked singularity for the self-similar case analyzed by Nolan and Mena [15].

We also study angular frequencies of physical fields which propagate along the null geodesics emanating from the central naked singularity. DJD showed that the redshift between the central naked singularity and any off-center observer is infinite [14]. Hence even if physical fields with finite frequencies are excited at the naked singularity, "physical" information of the naked singularity can not reach distant observers. However, it would not be natural that physical fields with finite frequencies are generated at the naked singularity where the energy density of the dust fluid and spacetime curvature diverge. In reality, linear perturbation analyses of LTB solution show that the spacetime curvature associated to gravitational-wave perturbations indefinitely grows in the limit to the Cauchy horizon $[16,17]$. This means that the frequencies of the linear gravitational waves generated at the naked singularity will be infinite even in purely classical dynamics. Physical fields excited by quantum effects also have infinite frequencies at the naked singularity [18-23]. Hence, in this article, we study the angular frequencies of the physical fields emanating from the central naked singularity with appropriately infinite frequencies.

This paper is organized as follows. In Sec.II, we present the model of background spacetime, which describes the gravitational collapse of a self-similar dust sphere with a finite mass. In Sec.III, we derive asymptotic behaviors of the null geodesics near the naked singularity and the basic equations for numerical calculations to see the intermediate behaviors of the null geodesics inside the dust sphere. In this section, we comment on the previous study about the topology of the naked singularity by Nolan and Mena. In Sec.IV, we explain the null geodesics in the exterior vacuum region. In Sec.V, we present the numerical results for 
the apparent size of the naked singularity, i.e., the angular diameter of the central naked singularity, or equivalently, the largest impact parameter of the singular null geodesic. In Sec.VI, the frequencies of physical fields propagating along null geodesics emanating from the central naked singularity are investigated. Finally, Sec.VII is devoted for summary and discussion.

In this article, we adopt the unit $G=c=\hbar=1$ and follow the sign conventions of the metric and Riemann tensors in Ref. [24].

\section{COLLAPSING DUST SPHERE}

In this section, we show the model of background spacetime; the gravitational collapse of a self-similar dust sphere with a finite mass. We assume that the dust fluid exists only within a finite comoving region $0 \leq r \leq r_{\mathrm{s}}$, where $r_{\mathrm{s}}$ is a positive constant.

\section{A. Interior Solution}

Dust fluid elements move along timelike geodesics. We can adopt the tangent vector of these geodesics as a temporal coordinate basis. Then the line element is written in the form

$$
d s^{2}=-d t^{2}+\frac{R^{2}(t, r) d r^{2}}{1+f(r)}+R^{2}(t, r)\left(d \theta^{2}+\sin ^{2} \theta d \varphi^{2}\right)
$$

where a prime denotes the derivative with respect to the comoving radial coordinate $r$, and $f(r)$ is an arbitrary function. In this coordinate system, the components of the 4-velocity of the dust fluid elements are $u^{\mu}=\delta_{t}^{\mu}$. Einstein equations and the equation of motion for the dust fluid lead to

$$
\begin{aligned}
\dot{R}^{2} & =\frac{F(r)}{R}+f(r), \\
\rho(t, r) & =\frac{F^{\prime}}{8 \pi R^{\prime} R^{2}},
\end{aligned}
$$

where a dot means the temporal derivative, $\rho(t, r)$ is the rest mass density of the dust fluid, and $F(r)$ is an arbitrary function. From Eq.(2.3), the arbitrary function $F(r)$ is regarded as 
the twice of a mass within the comoving radius $r$. Then Eq.(2.2) is regarded as an energy equation for a dust fluid element labeled by $r$ and hence $f(r)$ corresponds to the specific energy of it.

For simplicity, hereafter we assume the self-similarity for this spacetime; all the dimensionless variables are the functions of the self-similar variable $x:=t / r$ only. Then the arbitrary function $F(r)$ is written in the form

$$
F(r)=\Lambda_{0} r,
$$

where $\Lambda_{0}$ is a positive dimensionless constant. The specific energy $f(r)$ should vanish by this self-similar hypothesis. Then the solution of Eq.(2.2) is given in the form

$$
R=r\left(1-\frac{3 \sqrt{\Lambda_{0}}}{2} x\right)^{\frac{2}{3}}
$$

where using a freedom to re-scale the radial coordinate $r$, we have chosen an arbitrary function associated to the temporal integration so that $R=r$ at $t=0$. The dust fluid element at $r$ forms the shell focusing singularity at $t=2 r / 3 \sqrt{\Lambda_{0}}$.

\section{B. Exterior Solution}

The exterior region is assumed to be vacuum and hence is described by the Schwarzschild geometry by Birkhoff's theorem. Using the Schwarzschild static chart, the line element of the exterior region is written as

$$
d s^{2}=-C(R) d T^{2}+\frac{d R^{2}}{C(R)}+R^{2}\left(d \theta^{2}+\sin ^{2} \theta d \varphi^{2}\right),
$$

where the function $C(R)$ is defined by

$$
C(R):=1-\frac{2 M}{R}:=1-\frac{\Lambda_{0} r_{\mathrm{s}}}{R} .
$$

The angular coordinates $\theta$ and $\varphi$ are common to both the interior and exterior regions.

The trajectory of the surface $r=r_{\mathrm{s}}$ is a marginally bound timelike geodesic in the Schwarzschild spacetime by continuity. Thus the integration of the temporal component of geodesic equations and the normalization condition of the 4 -velocity vector lead to 


$$
\begin{aligned}
& \frac{d T}{d t}=\frac{1}{C(R)}, \\
& \frac{d R}{d t}=-\sqrt{\frac{2 M}{R}},
\end{aligned}
$$

where $t$ is the proper time which agrees with the interior temporal coordinate, and to derive the second equation, we have used the fact that the dust sphere is collapsing.

From Eqs.(2.8) and (2.9), the trajectory of the surface is determined by

$$
\frac{d R}{d T}=-C(R) \sqrt{\frac{2 M}{R}} .
$$

The above equation is easily integrated and we obtain

$$
T-T_{\mathrm{d}}=-\frac{2}{3} \sqrt{\frac{R}{2 M}}(R+6 M)-2 M \ln \left|\frac{\sqrt{R}-\sqrt{2 M}}{\sqrt{R}+\sqrt{2 M}}\right|,
$$

where $T_{\mathrm{d}}$ is an integration constant.

\section{NULL GEODESICS INSIDE THE DUST SPHERE}

By virtue of the spherical symmetry, it does not loose any generality to assume that the null geodesic is confined within the $\theta=\pi / 2$ plane and hence we do so. Then the tangent vector $k^{\mu}$ of the future directed outgoing null geodesic in the LTB spacetime is expressed in the form

$$
\begin{aligned}
k^{t} & =\frac{d t}{d \lambda}=: \frac{\mathcal{P}}{R}, \\
k^{r} & =\frac{d r}{d \lambda}=\frac{\sqrt{\mathcal{P}^{2}-l^{2}}}{R R^{\prime}}, \\
k^{\theta} & =\frac{d \theta}{d \lambda}=0, \\
k^{\varphi} & =\frac{d \varphi}{d \lambda}=\frac{l}{R^{2}},
\end{aligned}
$$

where $\lambda$ is the affine parameter and $l$ is an integration constant which corresponds to the

conserved angular momentum. Without loss of generality, we can assume $l \geq 0$. We call a null geodesic of $l=0$ the radial one while a null geodesic of $l>0$ is called the non-radial one. $\mathcal{P}$ should be larger than or equal to $l$ so that $k^{t}$ is positive and $k^{r}$ is real. In this article, 
we are interested in the null geodesics emanating from the central naked singularity; we call those the singular null geodesics.

Instead of the affine parameter $\lambda$, we adopt the comoving radius $r$ to parameterize the null geodesic. Then the geodesic equations become

$$
\begin{aligned}
r \frac{d \mathcal{P}}{d r} & =-\frac{1}{W^{4}} \sqrt{\Lambda_{0}\left(\mathcal{P}^{2}-l^{2}\right)} r^{2(1-\alpha)}+\frac{\mathcal{P}}{3 W^{3}}\left(W^{3}+2 r^{3(1-\alpha) / 2}\right) \\
\frac{d R}{d r^{\alpha}} & =\frac{1}{3 \alpha W^{2}}\left(W-\frac{\Lambda_{0}^{1 / 2} \mathcal{P}}{\sqrt{\mathcal{P}^{2}-l^{2}}} r^{(1-\alpha) / 2}\right)\left(W^{3}+2 r^{3(1-\alpha) / 2}\right)
\end{aligned}
$$

where we have introduced a new variable defined by

$$
W:=\sqrt{\frac{R}{r^{\alpha}}}
$$

and $\alpha$ is some positive constant which will be fixed in the following discussion.

\section{A. Asymptotic Behavior near the Singularity}

As discussed by Joshi and Dwivedi [10], singular null geodesics should behave near the singularity as

$$
R \longrightarrow W_{0}^{2} r^{\alpha} \quad \text { for } r \longrightarrow 0
$$

where $W_{0}$ is some positive constant. Assuming this behavior, we search for asymptotic

solutions of both the radial and non-radial singular null geodesics in the neighborhood of the central naked singularity.

\section{Radial Singular Null Geodesics}

The asymptotic behavior of the radial singular null geodesic have already been studied well by several researchers [9-12]. However, we would like to again see it in detail since the knowledge about the radial singular null geodesics is useful for the analysis of non-radial singular null geodesics.

For radial null geodesics, Eqs.(3.6) and (3.8) lead to 


$$
W_{0}^{2}=\lim _{r \rightarrow 0} \frac{1}{3 \alpha W_{0}^{2}}\left(W_{0}-\Lambda_{0}^{1 / 2} r^{(1-\alpha) / 2}\right)\left(W_{0}^{3}+2 r^{3(1-\alpha) / 2}\right)
$$

The above equation determines $W_{0}$ and $\alpha$. In order that the above equation has a positive root for $W_{0}, \alpha$ should be $1 / 3$ or unity. The solutions of $\alpha=1 / 3$ correspond to null geodesics from the origin before the formation of central singularity while the solutions of $\alpha=1$ are of our interest, i.e., the singular null geodesics. To see this fact, we rewrite Eq.(2.5) in the form

$$
t=\frac{2}{3 \Lambda_{0}^{1 / 2}}\left\{1-\left(\frac{R}{r}\right)^{3 / 2}\right\} r .
$$

Using the above equation and Eq.(3.8), we find that $t \rightarrow-2 W_{0}^{3} / 3 \Lambda_{0}^{1 / 2}$ for $r \rightarrow 0$ along the null geodesic of $\alpha=1 / 3$. This means that the null geodesics of $\alpha=1 / 3$ start from the regular center $r=0$ at $t<0$ and hence these are not singular null geodesics. On the other hand, in the case of $\alpha=1, t$ approaches to zero for $r \rightarrow 0$ along the null geodesic. Hence the null geodesics of $\alpha=1$ emanate from the central singularity $r=0$ at $t=0$.

Choosing $\alpha=1$, Eq.(3.9) leads to

$$
\Lambda_{0}^{1 / 2}=H\left(W_{0}\right):=\frac{2 W_{0}\left(1-W_{0}^{3}\right)}{W_{0}^{3}+2} .
$$

The radial singular null geodesics have to satisfy the above equation. Since $\Lambda_{0}^{1 / 2}$ is positive, $0<W_{0}<1$ should hold. We can easily find that there is a maximum of $H\left(W_{0}\right)$ at

$$
W_{0}=W_{\mathrm{m}}:=(-5+3 \sqrt{3})^{1 / 3}
$$

Hence in order that a singular null geodesic exists, $\Lambda_{0}$ should be less than or equal to a critical value $\Lambda_{\mathrm{m}}$ defined by

$$
\Lambda_{\mathrm{m}}:=H^{2}\left(W_{\mathrm{m}}\right)=\{4(26-15 \sqrt{3})\}^{2 / 3}
$$

We depict $H(y)$ in Fig.1. In this article, we will focus on the case in which $\Lambda_{0}$ is smaller than the critical value $\Lambda_{\mathrm{m}}$. Then form Fig.1, we can easily see that for a given value of $\Lambda_{0}$, there are two positive real roots of Eq.(3.11). We denote the smaller root by $W_{-}$and the other by $W_{\mathrm{c}}$. 
From Eq.(3.5), we find

$$
r \frac{d \mathcal{P}}{d r} \longrightarrow-\beta\left(W_{0}\right) \mathcal{P} \quad \text { for } r \longrightarrow 0
$$

where

$$
\beta\left(W_{0}\right):=\frac{2-10 W_{0}^{3}-W_{0}^{6}}{3 W_{0}^{3}\left(W_{0}^{3}+2\right)} .
$$

Since $\beta\left(W_{0}\right) \neq 0$ for $0<\Lambda_{0}<\Lambda_{\mathrm{m}}$, we obtain the asymptotic solution of $\mathcal{P}$ as

$$
\mathcal{P} \longrightarrow \text { Const. } \times r^{-\beta} \text {. }
$$

From the asymptotic behaviors of $W$ and $\mathcal{P}$, it is better to introduce the following variables,

$$
\begin{aligned}
P & :=r^{\beta} \mathcal{P}, \\
\Delta W & :=W-W_{0} .
\end{aligned}
$$

Since $\Delta W$ will vanish and $P$ approaches to some finite positive value $P_{0}$, for $r \rightarrow 0$, Eqs.(3.5) and (3.6) lead to

$$
\begin{aligned}
& \frac{d \Delta W}{d \ln r} \longrightarrow \beta \Delta W \\
& \frac{d P}{d \ln r} \longrightarrow \frac{2 P_{0}\left(2-5 W_{0}^{3}\right)}{W_{0}^{4}\left(W_{0}^{3}+2\right)} \Delta W
\end{aligned}
$$

Integrating the above equations, we obtain asymptotic solutions for $r \rightarrow 0$ as

$$
\begin{aligned}
& W \longrightarrow W_{0}+W_{* 0} r^{\beta}, \\
& \mathcal{P} \longrightarrow \frac{P_{0}}{r^{\beta}}\left\{1+\frac{6\left(2-5 W_{0}^{3}\right)}{W_{0}\left(2-10 W_{0}^{3}-W_{0}^{6}\right)} W_{* 0} r^{\beta}\right\},
\end{aligned}
$$

where $W_{* 0}$ is an integration constant.

Here again note that $W$ has to approach to $W_{0}$ in the limit of $r \rightarrow 0$. This means that $\beta$ should be positive as long as $W_{* 0}$ does not vanish. The positivity of $\beta$ leads to the following inequality

$$
0<W_{0}<W_{\mathrm{m}}
$$


Hence for non-vanishing $W_{* 0}$, the smaller root $W_{0}=W_{-}$of Eq.(3.11) has to be chosen (see Fig.1).

The other root $W_{\mathrm{c}}$ of Eq.(3.11) is larger than $W_{\mathrm{m}}$ and hence $\beta\left(W_{\mathrm{c}}\right)$ is negative. For such singular null geodesics, $W_{* 0}$ has to vanish so that $W$ approaches to $W_{\mathrm{c}}$ for $r \rightarrow 0$. This implies that $W$ should be always equal to $W_{\mathrm{c}}$ by Eq.(3.6). This null geodesic is a generator of the Cauchy horizon. Substituting $\alpha=1, l=0$ and $W=W_{\mathrm{c}}$ into Eq.(3.5), we can easily perform the integration and obtain the solution for $\mathcal{P}$ on the Cauchy horizon as

$$
\mathcal{P}=P_{\mathrm{c}} r^{-\beta_{\mathrm{c}}}
$$

where $P_{\mathrm{c}}$ is an integration constant and $\beta_{\mathrm{c}}:=\beta\left(W_{\mathrm{c}}\right)$. Since $\beta_{\mathrm{c}}<0, \mathcal{P}$ vanishes in the limit $r \rightarrow 0$.

\section{Non-Radial Singular Null Geodesics of $\mathcal{P} \rightarrow \infty$ for $r \rightarrow 0$}

Let us consider non-radial singular null geodesics of $\mathcal{P} \rightarrow+\infty$ for $r \rightarrow 0$. The argument from Eqs.(3.9) to (3.23) is almost completely applicable to this case. However, because $\mathcal{P}$ has to diverge for $r \rightarrow 0, \beta$ should be positive. Hence the smaller root $W_{0}=W_{-}$of Eq.(3.11) must be chosen.

The trajectory in the $(r, \varphi)$-plane is determined by the equation

$$
\frac{d \varphi}{d r}=\frac{l}{R^{2}} \frac{d \lambda}{d r}=\frac{l\left(W^{3}+2\right) r^{\beta_{-}-1}}{3 W^{3} \sqrt{P^{2}-l^{2} r^{2 \beta_{-}}}},
$$

where $\beta_{-}:=\beta\left(W_{-}\right)$. The above equation becomes for $r \rightarrow 0$ as

$$
\frac{d \varphi}{d r} \longrightarrow \frac{l\left(W_{-}{ }^{3}+2\right) r^{\beta_{-}-1}}{3 W_{-}{ }^{3} P_{0}} .
$$

Integrating the above equation, we find

$$
\varphi \longrightarrow \frac{l\left(W_{-}^{3}+2\right) r^{\beta_{-}}}{3 \beta_{-} W_{-}^{3} P_{0}}+\text { const. }
$$

Hence $\varphi$ has a finite limit for $r \rightarrow 0$. As discussed by Nolan and Mena [15], this result means that the singularity from which the non-radial null geodesics of $W_{0}=W_{-}$emanate is foliated by 2-sphere in accordance with Christodoulou's argument [25]. 


\section{Non-Radial Singular Null Geodesics with Finite $\left.\mathcal{P}\right|_{r=0}$}

Here, we investigate non-radial singular null geodesics with a finite limit of $\mathcal{P}$ for $r \rightarrow 0$. We denote this limit by $\mathcal{P}_{0}$ which should be larger than or equal to $l$. However, we can easily see that there is no consistent solution of the behavior, $\mathcal{P} \rightarrow l$ for $r \rightarrow 0$ (see Appendix A). Hence hereafter, we assume $\mathcal{P}_{0}>l$.

By the assumption, $\mathcal{P}$ is written in the form

$$
\mathcal{P}=\mathcal{P}_{0}+\delta \mathcal{P}(r)
$$

where $\delta \mathcal{P}$ satisfies

$$
\lim _{r \rightarrow 0} \delta \mathcal{P}=0
$$

Using Eqs.(3.8), (3.28) and (3.29), Eq.(3.6) becomes

$$
W_{0}^{2}=\lim _{r \rightarrow 0} \frac{1}{3 \alpha W_{0}^{2}}\left(W_{0}-\frac{\Lambda_{0}^{1 / 2} \mathcal{P}_{0}}{\sqrt{\mathcal{P}_{0}^{2}-l^{2}}} r^{\frac{1}{2}(1-\alpha)}\right)\left(W_{0}^{3}+2 r^{\frac{3}{2}(1-\alpha)}\right) .
$$

In order that the above equation has a positive root $W_{0}, \alpha$ should be $1 / 3$ or unity. By the same reason as in the previous cases, we find that $\alpha=1$ corresponds to the singular null geodesics, and hence we focus on it. Then the above equation leads to

$$
\Lambda_{0}^{1 / 2}=\frac{2 W_{0}\left(1-W_{0}^{3}\right) \sqrt{\mathcal{P}_{0}^{2}-l^{2}}}{\mathcal{P}_{0}\left(W_{0}^{3}+2\right)}
$$

Since $\Lambda_{0}^{1 / 2}$ is positive, $W_{0}$ is less than unity.

From Eq.(3.5), we find

$$
r \frac{d \mathcal{P}}{d r} \longrightarrow-\frac{\Lambda_{0}^{1 / 2}}{W_{0}^{2}} \sqrt{\mathcal{P}_{0}^{2}-l^{2}}+\frac{\mathcal{P}_{0}}{3 W_{0}^{3}}\left(W_{0}^{3}+2\right) \quad \text { for } r \longrightarrow 0 .
$$

By the assumption of $\mathcal{P} \rightarrow \mathcal{P}_{0}$, the right hand side of the above equation should vanish and hence following equation is obtained,

$$
\Lambda_{0}^{1 / 2}=\frac{\mathcal{P}_{0} W_{0}\left(W_{0}^{3}+2\right)}{3 \sqrt{\mathcal{P}_{0}^{2}-l^{2}}}
$$


From Eqs.(3.31) and (3.33), we obtain

$$
\mathcal{P}_{0}^{2}=\frac{6 l^{2}\left(1-W_{0}^{3}\right)}{2-10 W_{0}^{3}-W_{0}^{6}},
$$

Since the right hand side of the above equation has to be positive, we obtain the same inequality as Eq.(3.23). It is worthy to notice that if Eq.(3.23) holds, $\mathcal{P}_{0}^{2}$ is larger than $l^{2}$. Multiplying each side of Eqs.(3.31) and (3.33), we get

$$
\Lambda_{0}=I^{2}\left(W_{0}\right):=\frac{2}{3} W_{0}^{2}\left(1-W_{0}^{3}\right) .
$$

We also depict the function $I(y)$ in Fig.1. From this figure, we can easily see that there are two positive real roots of Eq.(3.35). Because of Eq.(3.23), we have to choose the smaller root which will be denoted by $W_{+}$. Fig.1 shows that $W_{+}$is always larger than $W_{-}$. Hence the singular null geodesics of $W_{0}=W_{+}$are in the past of the singular null geodesics of $W_{0}=W_{-}$in the sufficiently small neighborhood of the central naked singularity $r=0$.

By the assumption, we may rewrite $W$ in the form

$$
W=W_{+}+\delta W(r)
$$

where

$$
\lim _{r \rightarrow 0} \delta W=0 .
$$

Then in the limit of $r \rightarrow 0$, Eqs.(3.5) and (3.6) are approximated by the linearized equations with respect to $(\delta W, \delta \mathcal{P})$,

$$
\frac{d}{d \ln r}\left(\begin{array}{c}
\delta W \\
\delta \mathcal{P}
\end{array}\right)=\left(\begin{array}{ll}
M_{11} & M_{12} \\
M_{21} & M_{22}
\end{array}\right)\left(\begin{array}{c}
\delta W \\
\delta \mathcal{P}
\end{array}\right),
$$

where

$$
\begin{aligned}
& M_{11}=\frac{2-10 W_{+}{ }^{3}-W_{+}{ }^{6}}{3 W_{+}{ }^{3}\left(W_{+}{ }^{3}+2\right)}, \\
& M_{12}=\frac{\sqrt{2\left(1-W_{+}{ }^{3}\right)\left(2-10 W_{+}{ }^{3}-W_{+}{ }^{6}\right)^{3}}}{6 \sqrt{3} l W_{+}^{2}\left(W_{+}{ }^{3}+2\right)^{2}} \\
& M_{21}=\frac{2 l\left(2 W_{+}{ }^{3}+1\right) \sqrt{2\left(1-W_{+}{ }^{3}\right)}}{W_{+}^{4} \sqrt{3\left(2-10 W_{+}{ }^{3}-W_{+}{ }^{6}\right)}} \\
& M_{22}=-\frac{2-10 W_{+}{ }^{3}-W_{+}{ }^{6}}{3 W_{+}{ }^{3}\left(W_{+}{ }^{3}+2\right)} .
\end{aligned}
$$


Maiking the matrix $M_{i j}$ to be the diagonal form, Eq.(3.38) is written in the form,

$$
\frac{d}{d \ln r}\left(\begin{array}{c}
S_{1} \\
S_{2}
\end{array}\right)=\left(\begin{array}{cc}
\gamma & 0 \\
0 & -\gamma
\end{array}\right)\left(\begin{array}{c}
S_{1} \\
S_{2}
\end{array}\right)
$$

where

$$
\left(\begin{array}{c}
S_{1} \\
S_{2}
\end{array}\right)=\frac{1}{2 \gamma M_{12}}\left(\begin{array}{cc}
M_{11}+\gamma & M_{12} \\
-M_{11}+\gamma & -M_{12}
\end{array}\right)\left(\begin{array}{c}
\delta W \\
\delta \mathcal{P}
\end{array}\right),
$$

and

$$
\gamma:=\sqrt{-\operatorname{det} M}=\frac{\sqrt{\left(4-8 W_{+}{ }^{3}-5 W_{+}{ }^{6}\right)\left(2-10 W_{+}{ }^{3}-W_{+}{ }^{6}\right)}}{3 W_{+}{ }^{3}\left(W_{+}{ }^{3}+2\right)} .
$$

Since Eq.(3.23) should hold, $\gamma$ is real.

Solving Eq.(3.43), we obtain

$$
\left(\begin{array}{c}
S_{1} \\
S_{2}
\end{array}\right)=\left(\begin{array}{c}
C_{1} r^{+\gamma} \\
C_{2} r^{-\gamma}
\end{array}\right)
$$

where $C_{1}$ and $C_{2}$ are integration constants. Since $\delta W$ and $\delta \mathcal{P}$ vanish for $r \rightarrow 0, C_{2}$ should be equal to zero. Hence we find that for $r \rightarrow 0$,

$$
\begin{aligned}
& W \longrightarrow W_{+}+W_{\dagger 0} r^{\gamma}, \\
& \mathcal{P} \longrightarrow \mathcal{P}_{0}-\frac{M_{11}-\gamma}{M_{12}} W_{\dagger 0} r^{\gamma},
\end{aligned}
$$

where $W_{\dagger 0}$ is an arbitrary constant.

The asymptotic behavior of the trajectory in the $(r, \varphi)$-plane is determined by

$$
\frac{d \varphi}{d r}=\frac{l\left(W^{3}+2\right)}{3 r W^{3} \sqrt{\mathcal{P}^{2}-l^{2}}} \longrightarrow \frac{l\left(W_{+}{ }^{3}+2\right)}{3 r W_{+}{ }^{3} \sqrt{\mathcal{P}_{0}^{2}-l^{2}}} \quad \text { for } r \longrightarrow 0 .
$$

From the above equation, we obtain

$$
\varphi \longrightarrow \frac{l\left(W_{+}^{3}+2\right)}{3 W_{+}^{3} \sqrt{\mathcal{P}_{0}^{2}-l^{2}}} \ln r+\text { const. } \quad \text { for } r \longrightarrow 0 .
$$

Hence we find that $\varphi \rightarrow-\infty$ in the limit $r \rightarrow 0$. As discussed by Nolan and Mena [15], this means that the singularity from which the singular null geodesics of $W_{0}=W_{+}$emanate is topologically a "point" in the sense of Christodoulou [25]. As will be shown below, the singular null geodesics of $W_{0}=W_{+}$have the largest impact parameter at each moment. 


\section{B. The Basic Equations for Numerical Calculations}

In order to see the intermediate behavior of singular null geodesics inside the dust sphere, we numerically solve Eqs.(3.5) and (3.6) with $\alpha=1$ by the 4th order Runge-Kutta method. By virtue of the existence of the homothetic Killing vector, we obtain an analytic expression

for $\mathcal{P}$ as a function of $W$ (see Appendix B). However, we numerically integrate Eq.(3.5) and use the analytic expression for $\mathcal{P}$ to check the numerical accuracy. We will rewrite Eqs.(3.5) and (3.6) into appropriate forms for the numerical integrations below.

\section{Singular Null Geodesics of $\mathcal{P} \rightarrow \infty$ for $r \rightarrow 0$}

Here we consider singular null geodesics of $\mathcal{P} \rightarrow \infty$ for $r \rightarrow 0$, or equivalently $W_{0}=W_{-}$. From the asymptotic solutions (3.21) and (3.22), we introduce following variables,

$$
\begin{gathered}
w:=r^{\beta_{-},} \\
W_{*}(w):=w^{-1} \Delta W .
\end{gathered}
$$

Then Eqs.(3.5) and (3.6) are written in the form,

$$
\begin{aligned}
\frac{d P}{d w} & =\frac{2 W_{*}+\left(1+3 \beta_{-}\right) F_{1}+3 \Lambda_{0}^{1 / 2} w G_{1}}{3 \beta_{-}\left(W_{0}+w W_{*}\right)^{4}} P \\
\frac{d W_{*}}{d w} & =-\frac{2\left(1+3 \beta_{-}\right) W_{*} F_{2}+\left(2 W_{0}+\Lambda_{0}^{1 / 2}\right) F_{3}+\Lambda_{0}^{1 / 2}\left(W_{0}^{3}+2+w F_{2}\right) G_{2}}{6 \beta_{-}\left(W_{0}+w W_{*}\right)^{3}}
\end{aligned}
$$

where

$$
\begin{aligned}
F_{1} & :=4 W_{0}^{3} W_{*}+6 w W_{0}^{2} W_{*}^{2}+4 w^{2} W_{0} W_{*}^{3}+w^{3} W_{*}^{4}, \\
F_{2} & :=3 W_{0}^{2} W_{*}+3 w W_{0} W_{*}^{2}+w^{2} W_{*}^{3}, \\
F_{3} & :=3 W_{0} W_{*}^{2}+w W_{*}^{3} \\
G_{1} & :=\frac{l^{2}}{P\left(P+\sqrt{P^{2}-l^{2} w^{2}}\right)} \\
G_{2} & :=\frac{l^{2}}{\left(P+\sqrt{P^{2}-l^{2} w^{2}}\right) \sqrt{P^{2}-l^{2} w^{2}}} .
\end{aligned}
$$


Eqs.(3.53) and (3.54) can be easily integrated numerically from the origin $w=0$, or conversely from the surface $w=w_{\mathrm{s}}:=r_{\mathrm{s}}{ }^{\beta_{-}}$to $w=0$, since $w=0$ is not singular in these equations.

It should be noted that $W$ and $P$ is not uniquely determined by its initial value $W_{0}$ and $P_{0}$ only. Eq.(3.54) shows that the boundary value of $W_{*}$ is also necessary to determine them; we have denoted the value of $W_{*}(0)$ by $W_{* 0}$. Since $W_{0}$ should be equal to $W_{-}$, the free parameters to specify the solutions for $W$ and $P$ are $W_{* 0}, P_{0}$ and the conserved angular momentum $l$. However, here note that the solutions for $W$ constitute a two-parameter family with respect to $W_{* 0}$ and $l / P_{0}$. We can rewrite Eqs.(3.53) and (3.54) as the equations for $\hat{P}:=P / P_{0}$ and $W_{*}$. By introducing $\hat{l}:=l / P_{0}, P_{0}$ does not appear in these rewritten equations. The initial value of $\hat{P}$ is unity by definition. Hence the free parameters are $W_{* 0}$ and $\hat{l}$ in this new system of differential equations.

\section{Non-Radial Singular Null Geodesics with Finite $\left.\mathcal{P}\right|_{r=0}$}

We consider the case of $\mathcal{P} \rightarrow$ finite for $r \rightarrow 0$, or equivalently $W_{0}=W_{+}$. In this case, the conserved angular momentum $l$ is necessarily non-vanishing. From Eqs.(3.47) and (3.48), it is better to introduce following variables,

$$
\begin{gathered}
v:=r^{\gamma}, \\
W_{\dagger}(v):=v^{-1} \delta W, \\
\mathcal{P}_{\dagger}(v):=v^{-1} \delta \mathcal{P} .
\end{gathered}
$$

Then Eqs.(3.5) and (3.6) are written in the form,

$$
\begin{aligned}
\frac{d \mathcal{P}_{\dagger}}{d v} & =\frac{1}{3 \gamma W^{4} v^{2}}\left\{W\left(W^{3}+2\right)-3 \Lambda_{0}^{1 / 2} \sqrt{\mathcal{P}^{2}-l^{2}}-3 \gamma W^{4} \mathcal{P}_{\dagger} v\right\}, \\
\frac{d W_{\dagger}}{d v} & =\frac{1}{6 \gamma W^{3} v^{2}}\left\{2 W\left(1-W^{3}\right)-\frac{\Lambda_{0}^{1 / 2} \mathcal{P}\left(W^{3}+2\right)}{\sqrt{\mathcal{P}^{2}-l^{2}}}-6 \gamma W^{3} W_{\dagger} v\right\} .
\end{aligned}
$$

Eqs.(3.47) and (3.48) impose boundary conditions on $W_{\dagger}$ and $\mathcal{P}_{\dagger}$ as 


$$
\begin{aligned}
& W_{\dagger}(0)=W_{\dagger 0}, \\
& \mathcal{P}_{\dagger}(0)=\frac{\left(\gamma-M_{11}\right) W_{\dagger 0}}{M_{12}},
\end{aligned}
$$

where $W_{\dagger 0}$ is an arbitrary constant. The right hand sides of Eqs.(3.63) and (3.64) seem to be singular at $v=0$, but it is not true. Taking into account Eqs.(3.65) and (3.66), we can verify that the right hand sides have finite limits for $v \rightarrow 0$.

Once the initial value $W_{\dagger 0}$ is given, the initial value of $\mathcal{P}_{\dagger}$ is uniquely fixed by Eq.(3.66). Hence the solutions of $W_{\dagger}(v)$ constitute an one-parameter family with respect to $W_{\dagger 0}$ (the solutions of $W_{\dagger}$ seem to constitute a two-parameter family with respect to $W_{\dagger 0}$ and $l$, but it is not true). In order to see this fact, let us introduce following variables

$$
\begin{aligned}
& \tilde{\mathcal{P}}_{0}:=\frac{\mathcal{P}_{0}}{l}=\sqrt{\frac{6\left(1-W_{+}{ }^{3}\right)}{2-10 W_{+}{ }^{3}-W_{+}{ }^{6}}}, \\
& \tilde{\mathcal{P}}_{\dagger}:=\frac{\mathcal{P}_{\dagger}}{l}
\end{aligned}
$$

and rewrite Eqs.(3.63) and (3.64) into the equations for these variables. Then the parameter $l$ disappears in the new equations. This means that the solutions for $\tilde{\mathcal{P}}_{\dagger}$ and $W_{\dagger}$ do not depend

on $l$. Since $\tilde{\mathcal{P}}_{0}$ is not an arbitrary constant by Eq.(3.67), the remaining free parameter is the initial value $W_{\dagger 0}$ only.

\section{NULL GEODESICS IN THE EXTERIOR REGION}

The components of the null geodesic tangent on the equatorial plane $\theta=\pi / 2$ in the exterior Schwarzschild static chart are given by

$$
\begin{aligned}
k^{T} & =\frac{d T}{d \lambda}=\frac{\omega}{C(R)}, \\
k^{R} & =\frac{d R}{d \lambda}=\omega \sqrt{1-\frac{C(R)}{R^{2}}\left(\frac{l}{\omega}\right)^{2}}, \\
k^{\theta} & =\frac{d \theta}{d \lambda}=0, \\
k^{\varphi} & =\frac{d \varphi}{d \lambda}=\frac{l}{R^{2}},
\end{aligned}
$$


where $\omega$ and $l$ are integration constants which correspond to the angular frequency measured by the static observer at the future null infinity and to the conserved angular momentum, respectively.

In order to obtain a relation between the components of the interior and of the exterior regions, we introduce a tetrad basis whose components with respect to the interior LTB solution are given by

$$
\begin{aligned}
& \left(e_{(t)}^{\mu}\right)_{\mathrm{LTB}}=(1,0,0,0), \\
& \left(e_{(r)}\right)_{\mathrm{LTB}}=\left(0, \frac{1}{R^{\prime}}, 0,0\right), \\
& \left(e_{(\theta)}^{\mu}\right)_{\mathrm{LTB}}=\left(0,0, \frac{1}{R}, 0\right), \\
& \left(e_{(\varphi)}^{\mu}\right)_{\mathrm{LTB}}=\left(0,0,0, \frac{1}{R \sin \theta}\right) .
\end{aligned}
$$

Note that $e_{(t)}^{\mu}$ agrees with the 4 -velocity of the dust fluid element and $e_{(r)}{ }^{\mu}$ is normal to $e_{(t)}^{\mu}$ in $(t, r)$-space. At the surface of the dust sphere $r=r_{\mathrm{s}}$, the components of $e_{(t)}^{\mu}$ with respect to the exterior Schwarzschild static chart are obtained by Eqs.(2.8) and (2.9). The components of $e_{(r)}^{\mu}$ with respect to the exterior static chart are obtained as an outward normal to $e_{(t)}^{\mu}$ in $(T, R)$-space. Hence the components of the tetrad basis with respect to the exterior static chart are given by

$$
\begin{aligned}
& \left(e_{(t)}^{\mu}\right)_{\mathrm{Sch}}=\left(\frac{1}{C(R)},-\sqrt{\frac{2 M}{R}}, 0,0\right), \\
& \left(e_{(r)^{\mu}}\right)_{\mathrm{Sch}}=\left(\sqrt{\frac{2 M}{R}}, \frac{1}{C(R)}, 0,0\right), \\
& \left(e_{(\theta)}^{\mu}\right)_{\mathrm{Sch}}=\left(0,0, \frac{1}{R}, 0\right) \\
& \left(e_{(\varphi)}^{\mu}\right)_{\mathrm{Sch}}=\left(0,0,0, \frac{1}{R \sin \theta}\right) .
\end{aligned}
$$

Using the above expression, the continuities of $e_{(t)}{ }^{\mu} k_{\mu}$ and $e_{(r)}{ }^{\mu} k_{\mu}$ lead to the relation between the components in the exterior and in the interior coordinate systems at the surface of the dust sphere;

$$
k^{T}=\frac{1}{C(R)}\left(k^{t}-R^{\prime} \sqrt{\frac{2 M}{R}} k^{r}\right)
$$




$$
k^{R}=-\sqrt{\frac{2 M}{R}} k^{t}+R^{\prime} k^{r}
$$

Therefore, we obtain the angular frequency for the observer at the null infinity as

$$
\omega=\left(k^{t}-R^{\prime} \sqrt{\frac{2 M}{R}} k^{r}\right)_{r=r_{\mathrm{s}}} .
$$

The $\varphi$-components $k^{\varphi}$ in exterior and interior coordinate systems agree with each other and hence $l$ is common.

We consider the situation in which a distant static observer at some given areal radius $R=R_{\mathrm{o}}$ detects the null geodesics. There is the invariance of the time translation, $T \rightarrow T+$ const., in the exterior region. Using this symmetry, we set the origin of the time coordinate $T$ so that the observer intersects the Cauchy horizon at $T=0$. The future directed outgoing radial null geodesic in the exterior Schwarzschild region is determined by

$$
\frac{d T}{d R}=\frac{1}{C(R)}
$$

We can easily integrate the above equation and obtain

$$
T-T_{\mathrm{ng}}=R+2 M \ln |R-2 M|
$$

where $T_{\text {ng }}$ is an integration constant. Since the Cauchy horizon is generated by radial null geodesics which go through $R=R_{\mathrm{o}}$ at $T=0$, the trajectory of the Cauchy horizon should be written in the form

$$
T=T_{\mathrm{CH}}(R):=R-R_{\mathrm{o}}+2 M \ln \left|\frac{R-2 M}{R_{\mathrm{o}}-2 M}\right| .
$$

The Cauchy horizon intersects the surface of the dust sphere when its areal radius is given by

$$
R=R_{\mathrm{cs}}:=r_{\mathrm{s}} W_{\mathrm{c}}^{2}=\frac{2 M W_{\mathrm{c}}^{2}}{\Lambda_{0}}
$$

Hence the exterior time coordinate at the intersection $R=R_{\mathrm{cs}}$ is given by

$$
T=T_{\mathrm{cs}}:=T_{\mathrm{CH}}\left(R_{\mathrm{cs}}\right)
$$


The trajectory of the surface of the dust sphere is given by Eq.(2.11). The integration constant $T_{\mathrm{d}}$ in this equation is determined by the condition in which the areal radius of the dust surface is equal to $R_{\mathrm{cs}}$ at $T=T_{\mathrm{cs}}$. Then we obtain

$$
T_{\mathrm{d}}=T_{\mathrm{cs}}+\frac{2}{3} \sqrt{\frac{R_{\mathrm{cs}}}{2 M}}\left(R_{\mathrm{cs}}+6 M\right)+2 M \ln \left|\frac{\sqrt{R_{\mathrm{cs}}}-\sqrt{2 M}}{\sqrt{R_{\mathrm{cs}}}+\sqrt{2 M}}\right|,
$$

To obtain non-radial singular null geodesics in the exterior region, we integrate the null condition

$$
\frac{d T}{d R}=\frac{R}{C(R) \sqrt{R^{2}-(l / \omega)^{2} C(R)}}
$$

between the surface of the dust sphere $R=R_{\mathrm{s}}$ and the observer $R=R_{\mathrm{o}}$.

\section{APPARENT SIZE OF THE NAKED SINGULARITY}

First we consider the angle $\delta$ between the radial direction and the singular null geodesic at the observer $R=R_{\mathrm{o}}$ (see Fig.4). This angle $\delta$ is defined as follows. We introduce an orthonormal basis whose components with respect to the Schwarzschild static chart are given by

$$
\begin{aligned}
\left(e_{(T)}{ }^{\mu}\right)_{\mathrm{Sch}} & =\left(\frac{1}{\sqrt{C\left(R_{\mathrm{o}}\right)}}, 0,0,0\right), \\
\left(e_{(R)}{ }^{\mu}\right)_{\mathrm{Sch}} & =\left(0, \sqrt{C\left(R_{\mathrm{o}}\right)}, 0,0\right), \\
\left(e_{(\theta)}{ }^{\mu}\right)_{\mathrm{Sch}} & =\left(0,0, \frac{1}{R_{\mathrm{o}}}, 0\right) \\
\left(e_{(\varphi)}{ }^{\mu}\right)_{\mathrm{Sch}} & =\left(0,0,0, \frac{1}{R_{\mathrm{o}} \sin \theta}\right) .
\end{aligned}
$$

Then the angle $\delta$ is given by

$$
\tan \delta=\frac{e_{(\varphi)}{ }^{\mu} k_{\mu}}{e_{(R)}{ }^{\mu} k_{\mu}}=\frac{l}{\omega} \sqrt{\frac{C\left(R_{\mathrm{o}}\right)}{R_{\mathrm{o}}^{2}-(l / \omega)^{2} C\left(R_{\mathrm{o}}\right)}} .
$$

We can easily see that for sufficiently large $R_{\mathrm{o}}$, the angle $\delta$ becomes as

$$
\delta \longrightarrow \frac{b}{R_{\mathrm{O}}}
$$


where $b$ is a constant which is regarded as the impact parameter of the singular null geodesic with respect to the central naked singularity,

$$
b:=\lim _{R_{\mathrm{o}} \rightarrow \infty} R_{\mathrm{o}} \delta=\frac{l}{\omega}
$$

The angle $\delta$ depends on the location $R_{\mathrm{o}}$ of the observer. On the other hand, the impact parameter $b$ does not and hence it is more convenient to characterize the "size" of the naked singularity.

Eq.(4.2) is regarded as an energy equation of a test particle with a specific energy $1 / 2$,

$$
\frac{1}{2}\left(\frac{d R}{d \tilde{\lambda}}\right)^{2}+V(R ; b)=\frac{1}{2}
$$

where $\tilde{\lambda} \equiv \omega \lambda$ and

$$
V(R ; b) \equiv \frac{b^{2} C(R)}{2 R^{2}}
$$

The maximal value of the effective potential $V$ is $b^{2} / 54 M^{2}$ at $R=3 M$. Hence if the impact parameter $b$ is smaller than $3 \sqrt{3} M$, there is no forbidden region for the motion of the test particle and hence the null geodesic can escape to infinity if it is initially outgoing. On the other hand, in the case of $b \geq 3 \sqrt{3} M$, it is non-trivial whether the singular null geodesics can go away to the infinity. When $b$ is equal to $3 \sqrt{3} M$, the null geodesic of the circular orbit $R=3 M$ is possible although such an orbit is unstable.

Numerically integrating geodesic equations derived in Sec.III, we can draw trajectories in the $(w, W)$-plane. As an example, we depict the radial singular null geodesics of $W_{-}=$ $\sqrt{3 / 10}$ in Fig.2. By Eq.(3.11), $\Lambda_{0}$ is about 0.288. The radial singular null geodesic of $W_{0}=W_{-}$approaches to the Cauchy horizon $W=W_{\mathrm{c}}$ in the limit of $W_{* 0} \rightarrow \infty$. On the other hand, in Fig.3, we depict the trajectories of the non-radial singular null geodesics of $W_{0}=W_{ \pm}$. As in Fig.2, we choose $W_{-}=\sqrt{3 / 10}$. In this case, $W_{+}$is about 0.361. All the null geodesics in this figure are detected by the observer at $R_{\mathrm{o}}=100 \mathrm{M}$ at the moment $T=5 M$. Hence these null geodesics constitute an one-parameter family with respect to the impact parameter $b$. The lower most curve corresponds to the radial singular null geodesic 
$b=0$. There is a critical value $b_{\text {crit }}$ for the impact parameter $b\left(b_{\text {crit }}\right.$ is about $0.460 \times 3 \sqrt{3}$ in the case of this figure). The null geodesics of $b>b_{\text {crit }}$ do not start from $w=0$ and hence are not singular null geodesics; $W$ of these null geodesics becomes larger than $W_{\text {c }}$ near $w=0$. On the other hand, the null geodesics of $b \leq b_{\text {crit }}$ start from the singularity $w=0$. For $b<b_{\text {crit }}, W$ is equal to $W_{-}$at $w=0$, while in the case of $b=b_{\text {crit }}, W$ becomes $W_{+}$at $w=0$. Hence the singular null geodesic of $W_{0}=W_{+}$has the largest impact parameter(see also Fig.5).

The angular diameter $\Delta$ of the central naked singularity for an observer at $R=R_{\mathrm{o}}$ is the twice of the angle $\delta$ of the singular null geodesic with the largest impact parameter $b_{\text {crit }}$,

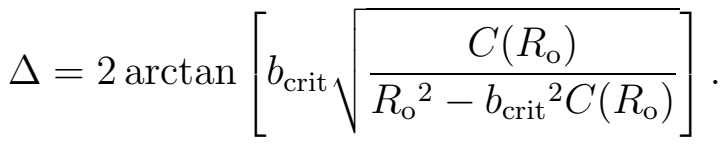

In order to study the temporal behavior of the largest impact parameter $b=b_{\text {crit }}$, we integrate Eqs.(3.63) and (3.64) from $v=0$ to $v=r_{\mathrm{s}}^{\gamma}$ for various boundary values $W_{\dagger 0}$. We set $l$ to be unity. After the integration to $v=r_{\mathrm{s}}^{\gamma}$ is completed, we obtain $\omega$ by Eq.(4.15); we denote $\omega$ of the null geodesic of $b=b_{\text {crit }}$ by $\omega_{\text {crit }}$. Then we integrate Eq.(4.22) until $R=R_{\mathrm{o}}$ and as a result, we obtain $T_{\mathrm{o}}$. Here note that $T_{\mathrm{o}}$ is a function of the initial value $W_{\dagger 0}$. We search for the initial value $W_{\dagger 0}$ so that some given $T_{\mathrm{o}}$ is realized as a result of the numerical integration. Then we obtain the largest impact parameter by $b_{\text {crit }}=\omega_{\text {crit }}$ for given $T_{\mathrm{o}}$.

In Fig.6, we depict the largest impact parameter $b_{\text {crit }}$ as a function of the exterior time coordinate $T_{\mathrm{o}}$ at the observer $R_{\mathrm{o}}=100 M$. Since $R_{\mathrm{o}}$ is sufficiently larger than $2 M$, the angular diameter $\Delta$ of the central naked singularity is almost equal to $2 b_{\text {crit }} / R_{\mathrm{o}}$.

From Fig.6, we see that the largest impact parameter $b_{\text {crit }}$ approaches to $b_{\mathrm{B}}:=3 \sqrt{3} M$ asymptotically. Hence our numerical results strongly suggest that the largest impact parameter $b_{\text {crit }}$ is bounded above by $b_{\mathrm{B}}$. The angular diameter $\Delta$ is also bounded above by

$$
\Delta_{\mathrm{B}}=2 \arctan \left[b_{\mathrm{B}} \sqrt{\frac{C\left(R_{\mathrm{o}}\right)}{R_{\mathrm{o}}^{2}-b_{\mathrm{B}}^{2} C\left(R_{\mathrm{o}}\right)}}\right] .
$$

First, at the Cauchy horizon $T_{\mathrm{o}}=0$, the central naked singularity is observed as a point, i.e., $b_{\text {crit }}=0$, or equivalently $\Delta=0$. Then gradually the largest impact parameter $b_{\text {crit }}$ grows 
and approaches to $b_{\mathrm{B}}$; correspondingly, the angular diameter approaches to $\Delta_{\mathrm{B}}$.

\section{FREQUENCIES OF PHYSICAL FIELDS FROM THE NAKED SINGULARITY}

The redshift $z_{0}$ between the central naked singularity and a timelike observer with a 4-velocity $v^{\mu}$ is given by

$$
z_{0}+1 \equiv \frac{1}{k_{\mu} v^{\mu}} \lim _{r \rightarrow 0} k_{\nu} u^{\nu}
$$

Since $k_{\nu} u^{\nu}=-\mathcal{P} / R \rightarrow-\infty$ for $r \rightarrow 0$ for finite $k_{\mu} v^{\mu}$, the redshift $z_{0}$ is infinite. From this fact, it seems to be likely that physical information of the naked singularity cannot arrive at any observer. However it may not be necessarily true. Since the energy density and spacetime curvature are infinite at the naked singularity, physical fields generated there might also have infinite frequencies. Hence even if the physical fields suffer the infinite redshift, it is a non-trivial issue whether their frequencies observed at infinity vanish or not.

We do not exactly know what and how amount of physical fields are generated in the neighborhood of the central naked singularity where quantum effects of gravity might be important. However we may say that the physical field which can arrive at the distant observer from the dense region around the naked singularity might be graviton due to the weakness of its coupling to other fields. Further, it might not be so terrible to assume that

the frequency of the graviton is order of $\sqrt{\rho}$ which is the inverse of the free fall time. From Eq.(2.3), along a singular null geodesic, the density $\rho$ behaves as

$$
\rho \longrightarrow \frac{3 W_{0}^{3}\left(1-W_{0}^{3}\right)^{2}}{2 \pi\left(W_{0}^{3}+2\right)^{3}} \times \frac{1}{R^{2}} \quad \text { for } r \longrightarrow 0 .
$$

Hence hereafter we will focus on a physical field which is generated at $R=L$ with a comoving angular frequency $\omega_{L}=1 / L$. Of course, $L$ is assumed to be sufficiently smaller than $M$. It should be noted that the angular frequency of the physical field propagating along such a null geodesic becomes infinite at the central naked singularity. 


\section{A. On the Cauchy Horizon}

First, let us consider the angular frequency of the physical field which propagates on the Cauchy horizon. As mentioned, the Cauchy horizon is $R=W_{\mathrm{c}}^{2} r$. Hereafter we will denote the comoving radius of the singular null geodesic at $R=L$ by $r_{L}$. Then $r_{L}$ is equal to $L / W_{\mathrm{c}}^{2}$ on the Cauchy horizon. From Eq.(3.24), the angular frequency at $R=L$ is given by

$$
\omega_{L}=\frac{P_{\mathrm{c}} W_{\mathrm{c}}^{2 \beta_{\mathrm{c}}}}{L^{\beta_{\mathrm{c}}+1}}
$$

As mentioned, we assume that the angular frequency $\omega_{L}$ is $1 / L$ at $R=L$ and hence from the above equation, we obtain

$$
P_{\mathrm{c}}=\left(\frac{L}{W_{\mathrm{c}}^{2}}\right)^{\beta_{\mathrm{c}}}
$$

From Eq.(4.15), the angular frequency for a static observer at infinity is given by

$$
\begin{aligned}
\omega & =\left[k^{t}-R^{\prime} \sqrt{\frac{2 M}{R}} k^{r}\right]_{r=r_{\mathrm{s}}} \\
& =\frac{1}{2 M}\left(\frac{L}{2 M}\right)^{\beta_{\mathrm{c}}}\left\{\frac{4 W_{\mathrm{c}}\left(1-W_{\mathrm{c}}^{3}\right)^{2}}{\left(W_{\mathrm{c}}+2\right)^{2}}\right\}^{\beta_{\mathrm{c}}+1}\left\{1+\frac{2 W_{\mathrm{c}}^{1 / 2}\left(1-W_{\mathrm{c}}^{3}\right)}{W_{\mathrm{c}}+2}\right\} .
\end{aligned}
$$

It should be noted that since $\beta_{\mathrm{c}}$ is negative, $\omega$ diverges in the limit of $L \rightarrow 0$.

We find that in the limit of $W_{\mathrm{c}} \rightarrow 1$, the angular frequency $\omega$ behaves as

$$
\omega \longrightarrow \frac{1}{L}
$$

This is because $W_{\mathrm{c}}=1$ corresponds to no dust-sphere case $\Lambda_{0}=0$. Hence in the case of sufficiently small $\Lambda_{0}$, the angular frequency $\omega$ observed at the infinity is almost equal to the initial value $\omega_{L}$ at $R=L$. However it should be noted that small $\Lambda_{0}$ does not necessarily

corresponds to small total mass $M$. Because of $M=\Lambda_{0} r_{\mathrm{s}}$, if the comoving radius $r_{\mathrm{s}}$ of the dust surface is very large, $M$ can also be large. On the other hand, in the limit of $W_{\mathrm{c}} \rightarrow W_{\mathrm{m}}$, the angular frequency becomes as

$$
\omega \longrightarrow \frac{\Lambda_{\text {crit }}}{2 M W_{\mathrm{m}}}\left\{1+\left(\frac{\Lambda_{\text {crit }}}{W_{\mathrm{m}}}\right)^{1 / 2}\right\} \sim 0.243 M^{-1} .
$$

The angular frequency $\omega$ does not depend on $L$ in this limit. 


\section{B. In the Future of the Cauchy Horizon}

Now let us consider singular null geodesics in the future of the Cauchy horizon. The angular frequency of the physical field propagating along the singular null geodesic of the largest impact parameter $b=b_{\text {crit }}$ is obtained by integrating Eqs.(3.63) and (3.64) from $v=0$ to $v=r_{\mathrm{s}}{ }^{\gamma}$. For the exterior region, we integrate Eq.(4.22) until $R$ becomes some given $R_{\mathrm{o}}$ to obtain $T$ at $R=R_{\mathrm{o}}$. Then the initial value of $W_{\dagger}$ is determined iteratively so that $T$ becomes some given $T_{\mathrm{o}}$ at $R=R_{\mathrm{o}}$. The initial value of $\mathcal{P}$ is unity, i.e., $\mathcal{P}_{0}=1$, in order that $\omega_{L}=1 / L$. This condition and Eq.(3.34) lead to

$$
l=l_{\text {crit }}:=\sqrt{\frac{2-10 W_{+}{ }^{3}-W_{+}{ }^{6}}{6\left(1-W_{+}{ }^{3}\right)}} .
$$

As a result of the numerical integration, we obtain the angular frequency $\omega_{\text {crit }}$ and the impact parameter $b_{\text {crit }}=l_{\text {crit }} / \omega_{\text {crit }}$. Here note that the angular frequency $\omega_{\text {crit }}$ does not depend on the assumed value of $L$.

In order to study the angular frequencies for the distant static observer in the case of the null geodesics of $W_{0}=W_{-}$, we had better integrating the geodesic equations from the observer toward the central naked singularity. First, we integrate Eq.(4.22) from the observer $R=R_{\mathrm{o}}$ toward the dust sphere $R=R_{\mathrm{s}}$. The initial condition is the given $T_{\mathrm{o}}$. We set $\omega$ to be unity and hence the impact parameter is equal to $l$ which should be smaller than $b_{\text {crit }}$

obtained in the above. After the null geodesic reaches $R=R_{\mathrm{s}}$, we integrate Eqs.(3.53) and (3.54) toward the central naked singularity $w=0$. The initial condition for these equations are obtained by Eqs.(4.13) and (4.14) as

$$
P\left(w_{\mathrm{s}}\right)=\frac{w_{\mathrm{s}} R_{\mathrm{s}}}{C\left(R_{\mathrm{s}}\right)}\left[1+\sqrt{\frac{2 M}{R}\left\{1-\frac{l^{2} C\left(R_{\mathrm{s}}\right)}{\omega^{2} R_{\mathrm{s}}^{2}}\right\}}\right],
$$

and

$$
W_{*}\left(w_{\mathrm{s}}\right)=\frac{1}{w_{\mathrm{s}}}\left(\sqrt{\frac{\Lambda_{0} R_{\mathrm{s}}}{2 M}}-W_{-}\right) .
$$

Once we obtain $P$ at $R=L$, we obtain the observed angular frequency $\omega_{\mathrm{o}}$ for the static observer at $R=R_{\mathrm{o}}$ as 


$$
\omega_{\mathrm{o}}=\frac{w_{L}}{P\left(w_{L}\right) \sqrt{C\left(R_{\mathrm{o}}\right)}}
$$

where $w_{L}=r_{L}{ }^{\beta_{-}}$.

In Figs.7 and 8, we depict the angular frequency $\omega_{\mathrm{o}}$ for the static observer at $R_{\mathrm{o}}=100 \mathrm{M}$ as a function of the impact parameter $b$. Fig.7 corresponds to the case of $L=10^{-2} M$ while Fig. 8 is for $L=10^{-3} M$. Since $R_{\mathrm{o}}$ is sufficiently large, the angle $\delta$ is almost equal to $b / R_{\mathrm{o}}$.

The background model is chosen so that $W_{-}=\sqrt{3 / 10}$. There are four curves; these are the data at $T_{\mathrm{o}}=5 M, 10 M, 15 M$ and $20 M$, respectively. The largest impact parameter $b_{\text {crit }}$ normalized by $b_{\mathrm{B}}$ for each moment is about $0.460,0.736,0.882$ and 0.951 , respectively. The angular frequency is monotonically increasing with respect to the impact parameter $b$ or equivalently, the angle $\delta$, while it monotonically decreases with the lapse of time. The angular frequency at $b=0$ will vanish in the limit of $T_{\mathrm{o}} \rightarrow \infty$, since in this limit, the radial null geodesic agrees with the generator of the event horizon whose redshift is infinite.

The angular frequency $\omega_{\text {crit }}$ is depicted as a function of the time $T_{\mathrm{o}}$ in Fig.9. This figure suggests that $\omega_{\text {crit }}$ approach to some finite value in the limit of $T \rightarrow \infty$. Hence the naked singularity will be observed as a ring for sufficiently late time.

\section{SUMMARY AND DISCUSSION}

We studied non-radial null geodesics emanating from the central naked singularity formed by the gravitational collapse of a self-similar dust sphere. The existence of the non-radial singular null geodesics means that the central naked singularity is observed as a disk if it emits null geodesics of all possible impact parameters. The observed singular null geodesic with the largest impact parameter $b=b_{\text {crit }}$ gives the angular diameter $\Delta$ of the naked singularity. The angular diameter $\Delta$ is time dependent; it monotonically grows and approaches to the value $3 \sqrt{3} M / R_{\mathrm{o}}$ for the observer at $R=R_{\mathrm{o}} \gg M$. The asymptotic value of the angular diameter comes from the geometry of the exterior Schwarzschild region.

As shown by Nolan and Mena [15], in the case of the self-similar LTB solution, the topology of the central naked singularity is not uniform, being point-like on one region 
and spherical on another. In this article, we have shown that the point-like region of the central naked singularity is a portion from which singular null geodesics of the largest impact parameter $b=b_{\text {crit }}$ emanates. In other words, the outer boundary of the observed disk constitutes the null geodesics from the point-like portion of the central naked singularity. On the other hand, all the other singular null geodesics emanate from the spherical region of the central naked singularity.

We also studied the angular frequencies of the physical fields which propagate along the singular null geodesics. It is well known fact that the redshift between this naked singularity and any timelike observer is infinite. Hence even if some physical field with a finite frequency is excited at the central naked singularity, it cannot carry its energy from the central naked singularity to the outside. From this result, it seems to be likely that no physical information comes form the central naked singularity to any observer. However, it might be terrible to assume that some physical field with a finite frequency are generated at the naked singularity at which the energy density of the dust fluid and spacetime curvature are infinite. Graviton might be only one physical field which can carry the physical information to distant observers from the central naked singularity since it might not be scattered nor absorbed even in the situation with the extremely high energy density like as in the neighborhood of the central naked singularity. Its frequency might be order of $\sqrt{\rho}$, that is, the inverse of the free fall time. Since the energy density $\rho$ near the central naked singularity is proportional to $1 / R^{2}$ along the singular null geodesic, the angular frequency of this field generated at $R=L$ are order of $1 / L$. Hence in this article, we have considered the null geodesics which start at $R=L$ with the comoving angular frequency $\omega_{L}=1 / L$ and then have investigated the angular frequency $\omega_{\mathrm{o}}$ observed at $R=R_{\mathrm{o}} \gg M$. On the ground of dimensional analysis, if the fields are excited by the quantum gravitational effects, $L$ will be order of the Planck length.

As a result, we found that the angular frequency is a function of the impact parameter $b$, or equivalently the angle $\delta$ between the radial direction and the null geodesic. At the center $\delta=0$, the angular frequency $\omega$ is the smallest, while it is the largest at the boundary 
$\delta=\Delta / 2$. Hence, if such null geodesics are really emitted, the naked singularity is observed as a disk-like rainbow.

In the limit of $T \rightarrow \infty$, the angular frequency $\omega$ at $\delta=0$ vanishes while it does not for $0<\delta \leq \Delta / 2$. The singular null geodesic at $\delta=0$ in the limit of $T \rightarrow \infty$ is the generator of the event horizon and hence suffers the infinite gravitational redshift. By virtue of this gravitational redshift of the central naked singularity, we might observe the gravitons in a wide range of the angular frequency. Hence all the gravitational wave detectors (TAMA [26], LIGO [27], VIRGO [28], GEO [29], LISA [30] and DECIGO [31]) now in operation or in plan are able to detect the graviton from this naked singularity if sufficient amount of gravitons are excited there.

There are several researches for more general spherically symmetric systems and those revealed that non-vanishing pressure does not necessarily prevent the formation of the central shell focusing naked singularity [32-35]. In these example, there might be non-radial null geodesics emanating from the central naked singularity. The investigation of these is future work.

\section{Acknowledgements}

We are grateful to T. Harada and the colleagues in Department of Physics, Osaka City University for useful discussion. KN also thanks to the workshop of DECIGO held in National Astronomical Observatory of Japan in May, 2002.

\section{APPENDIX A}

In this appendix, we show that there is no singular null geodesic of $\mathcal{P} \rightarrow l$ for $r \rightarrow 0$ in the self-similar LTB spacetime.

Proof. First we assume $\mathcal{P} \rightarrow l$ in the limit $r \rightarrow 0$. Hence, $\mathcal{P}$ is written in the form

$$
\mathcal{P}=l+\delta \mathcal{P}(r),
$$

where $\delta \mathcal{P}$ satisfies 


$$
\lim _{r \rightarrow 0} \delta \mathcal{P}=0
$$

Then in the limit $r \rightarrow 0$, Eq.(3.6) becomes

$$
W_{0}^{2}=\lim _{r \rightarrow 0} \frac{1}{3 \alpha W_{0}^{2}}\left\{W_{0}-\sqrt{\frac{l \Lambda_{0} r^{1-\alpha}}{2 \delta \mathcal{P}}}\right\}\left\{W_{0}^{3}+2 r^{\frac{3}{2}(1-\alpha)}\right\} .
$$

Since the right hand side of the above equation should be finite, $\alpha$ is less than unity. Therefore, in the limit of $r \rightarrow 0$, Eq.(3.5) leads to

$$
\frac{d \delta \mathcal{P}}{d \ln r} \longrightarrow \frac{l}{3}
$$

Integrating the above equation, we obtain

$$
\delta \mathcal{P} \longrightarrow \frac{l}{3} \ln r \quad \text { for } r \longrightarrow 0
$$

This behavior is in contradiction to the assumption (A2).

\section{APPENDIX B}

In the self-similar LTB solution, there is a homothetic Killing vector $\xi^{a}$ which satisfies

$$
£_{\xi} g_{a b}=g_{a b}
$$

In this spacetime, its components are given by

$$
\xi^{\mu}=(t, r, 0,0)
$$

We can easily see that $C_{\xi} \equiv-k^{\mu} \xi_{\mu}$ is constant along a null geodesic with tangent $k^{\mu}$. By virtue of this fact, we obtain an analytic expression for $\mathcal{P}[9]$.

The self-similar variable $x$ is expressed by $W$ as

$$
x=\frac{2}{3 \Lambda_{0} 1 / 2}\left(1-W^{3}\right)
$$

Hence $R^{\prime}$ can be also expressed by $W$ as

$$
R^{\prime}=W^{2}+r\left(\partial_{r} W^{2}\right)_{t}=\frac{W^{3}+2}{3 W} .
$$


The conserved quantity associated with the homothetic Killing vector is

$$
C_{\xi}=t k^{t}-r R^{\prime 2} k^{r}
$$

From the null condition $k^{\mu} k_{\mu}=0$ and Eq.(B5), we obtain for non-vanishing $C_{\xi}$,

$$
\mathcal{P}=\frac{C_{\xi} W^{2}}{x^{2}-R^{\prime 2}}\left\{x \pm R^{\prime} \sqrt{1-\left(x^{2}-R^{\prime 2}\right) l^{2} /\left(C_{\xi} W^{2}\right)^{2}}\right\}
$$

When $C_{\xi}=0$ but $l$ does not vanish, the positive root of $\mathcal{P}$ is

$$
\mathcal{P}=\frac{l R^{\prime}}{\sqrt{R^{\prime 2}-x^{2}}} .
$$

For the case of $C_{\xi}=0=l$, the solution of Eq.(3.54) is $W_{*}=0$. Using this solution, Eq.(3.53) can be easily integrated as

$$
\mathcal{P}=\frac{P_{0}}{w}
$$

We numerically integrate Eqs.(3.53) and (3.63). Then the solutions (B6), (B7) and (B8) are used to check the accuracy of the numerical integrations. In our numerical calculations, the relative error estimated by using these analytic solutions for $\mathcal{P}$ is less than $10^{-7}$. 


\section{REFERENCES}

[1] R. Penrose, Riv. Nuovo Cim. 1, 252 (1969).

[2] R. Penrose, Phys. Rev. Lett. 14, 57 (1965).

[3] S. W. Hawking, Proc. R. Soc. London A300, 187 (1967).

[4] S. W. Hawking and R. Penrose, Proc. R. Soc. London A314, 529 (1970).

[5] S. W. Hawking, Commun. Math. Phys. 25, 152 (1972).

[6] D. M. Eardley and L. Smarr, Phys. Rev. D19, 2239 (1979).

[7] D. Christodoulou, Commun. Math. Phys. 93, 171 (1984).

[8] R. P. A. C. Newman, Class. Quantum Grav. 3, 527 (1986).

[9] P. S. Joshi and I. H. Dwivedi, Commun. Math. Phys. 146, 333 (1992).

[10] P. S. Joshi and I. H. Dwivedi, Phys. Rev. D45, 5357 (1993).

[11] T.P. Singh and P.S. Joshi, Class. Quantum Grav. 13, 559 (1996).

[12] S. Jhingan, P.S. Joshi and T.P. Singh, Class. Quantum Grav. 13, 3057 (1996).

[13] F. C. Mena and B. C. Nolan, Class. Quantum Grav. 18, 4531 (2001).

[14] S. S. Deshingkar, P. S. Joshi and I. H. Dwivedi, Phys. Rev. D65, 084009 (2002).

[15] B. C. Nolan and F. C. Mena, Class. Quantum Grav. 19, 2587 (2002).

[16] H. Iguchi, T. Harada and K. Nakao, Prog. Theor. Phys. 103, 53 (2000).

[17] K. Nakao, H. Iguchi and T. Harada, Phys. Rev. D63, (2001) 084003.

[18] S. Barve, T.P. Singh, C. Vaz and L. Witten, Nucl. Phys. B532, (1998) 361.

[19] S. Barve, T.P. Singh, C. Vaz and L. Witten, Phys. Rev. D58, 104018 (1998).

[20] C. Vaz and L. Witten, Phys. Lett. B442, 90 (1998). 
[21] T. Harada, H. Iguchi and K. Nakao, Phys. Rev. D61, 101502(R) (2000).

[22] T. Harada, H. Iguchi and K. Nakao, Phys. Rev. D62, 084037 (2000).

[23] T. Harada, H. Iguchi, K. Nakao, T. P. Singh, Takahiro Tanaka and Cenalo Vaz, Phy. Rev. D64, (2001), pp.041501(R)-1-5.

[24] R. M. Wald, General Relativity (The University of Chicago Press, 1984).

[25] D. Christodoulou, Ann. Math. 140, 607 (1984).

[26] S. Nagano, Proc. 2nd TAMA Workshop (Tokyo, Japan, 1999): Gravitational Wave Detection II, eds. S. Kawamura and N. Mio (2000) p.89; M. Ando, ibid., p.101; D. Tatsumi et al., ibid., p.113; S. Telada et al., ibid., p.129; N. Kanda et al., ibid., p.137; H. Tagoshi et al., ibid., p.145; R. Takahashi, ibid., p.151.

[27] A. Abramovici et al., Science 256, 325 (1992).

[28] C. Bradaschia et al., Nucl. Instrum. and Methods A289, 518 (1990).

[29] J. Hough, in Proceedings of Sixth Marcel Grossman Meeting, ed. by H. Sato and T. Nakamura (World Scientific, Singapore, 1992), p.192.

[30] LISA Science Team, Laser Interferometer Space Antenna for the detection and observation of gravitational waves: Pre-Phase A Report, Dec. 1995.

[31] N. Seto, S. Kawamura and T. Nakamura, Phys. Rev. Lett. 87, 221103 (2001).

[32] A. Ori and T. Piran, Gen. Relativ. Gravit. 20, 7 (1988).

[33] A. Ori and T. Piran, Phys. Rev. D42, 1068 (1990).

[34] T. Harada, Phys. Rev. D58, 104015 (1998).

[35] T. Harada, H. Iguchi and K. Nakao, Phys. Rev. D58, 104015 (1998). 


\section{FIGURES}

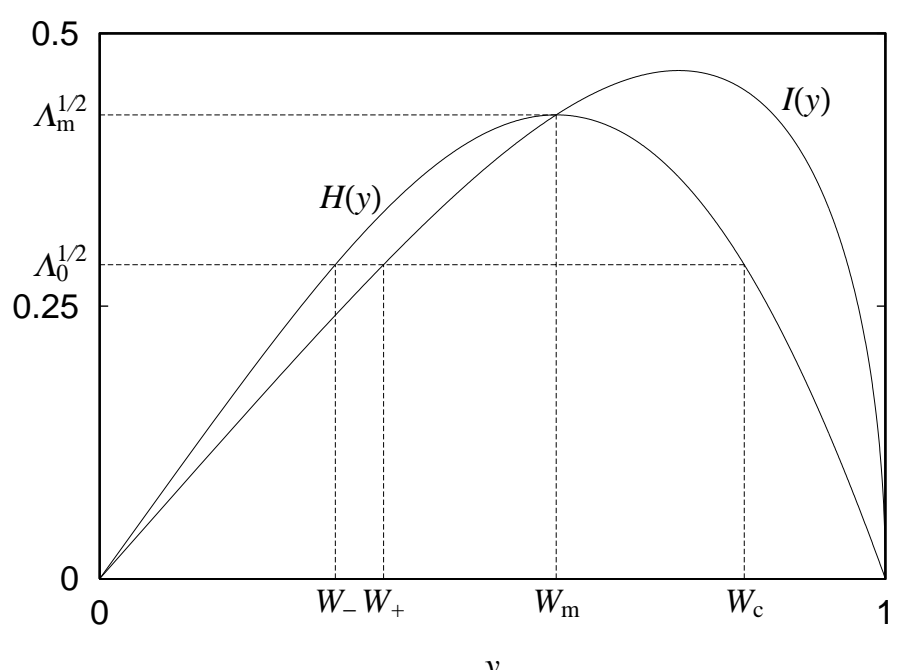

FIG. 1. The functions $H(y)$ and $I(y)$ are depicted. The roots of Eqs.(3.9) and (3.11) are also depicted for a given $\Lambda_{0}$ which is only one free parameter of the self-similar dust sphere.

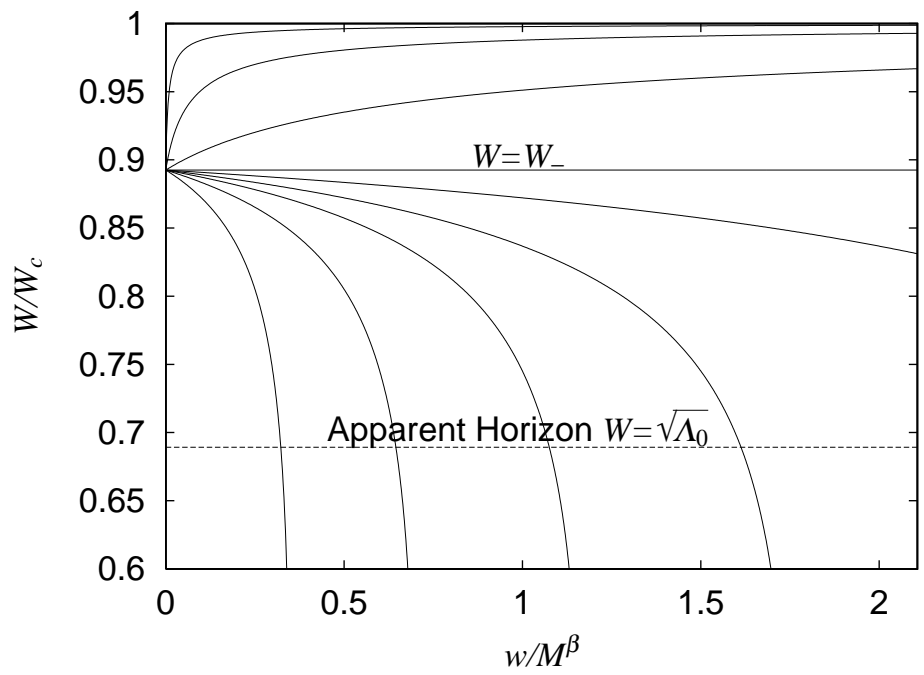

FIG. 2. The trajectories of radial singular null geodesics $l=0$ are depicted in $(w, W)$-plane.

From this figure, we can see that in the limit of $W_{* 0} \rightarrow \infty$, the singular null geodesic approaches to the Cauchy horizon $W=W_{\mathrm{c}}$. We also plot the apparent horizon $F / R=1$, or equivalently $W=\sqrt{\Lambda_{0}}$ in this plane. 


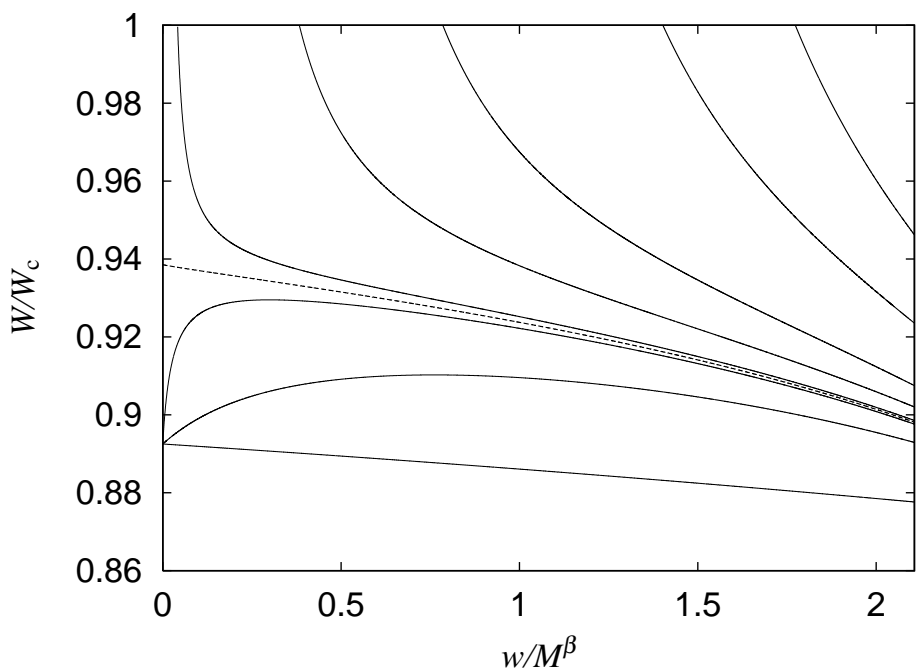

FIG. 3. The trajectories of singular null geodesics with various impact parameters $b$ are depicted in $(w, W)$-plane. These null geodesics intersect with each other at $(T, R)=(5 M, 100 M)$ in the exterior region. The lower most curve corresponds to the radial null geodesic $l=0(b=0)$. The dashed curve is the non-radial singular null geodesic with the largest impact parameter $b=b_{\text {crit }}$. The solid curves below this dashed curve are all the singular null geodesics while those above this curve are not.

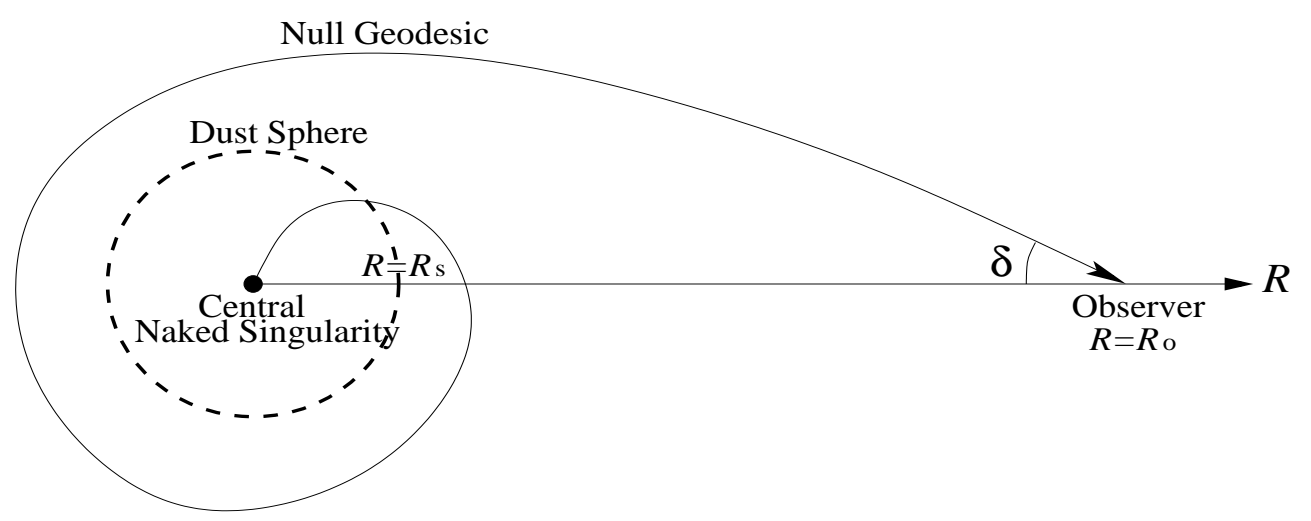

FIG. 4. The schematic diagram of the trajectory of a non-radial singular null geodesic. 


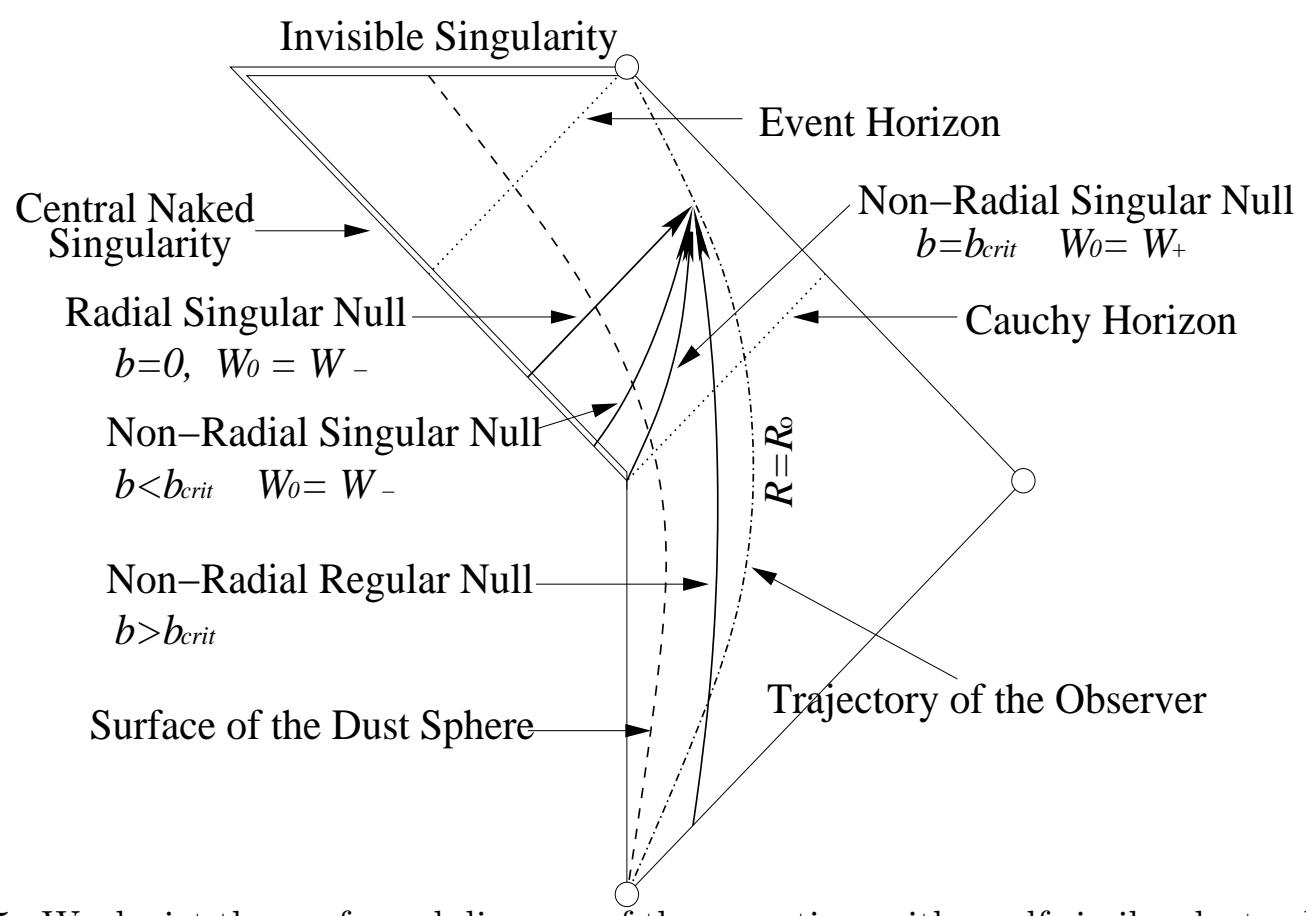

FIG. 5. We depict the conformal diagram of the spacetime with a self-similar dust sphere. The singular null geodesic of $b=b_{\text {crit }}$ emanates from the beginning of Cauchy horizon in this diagram. The observed null geodesics of $b>b_{\text {crit }}$ do not go through the origin $r=0$. 


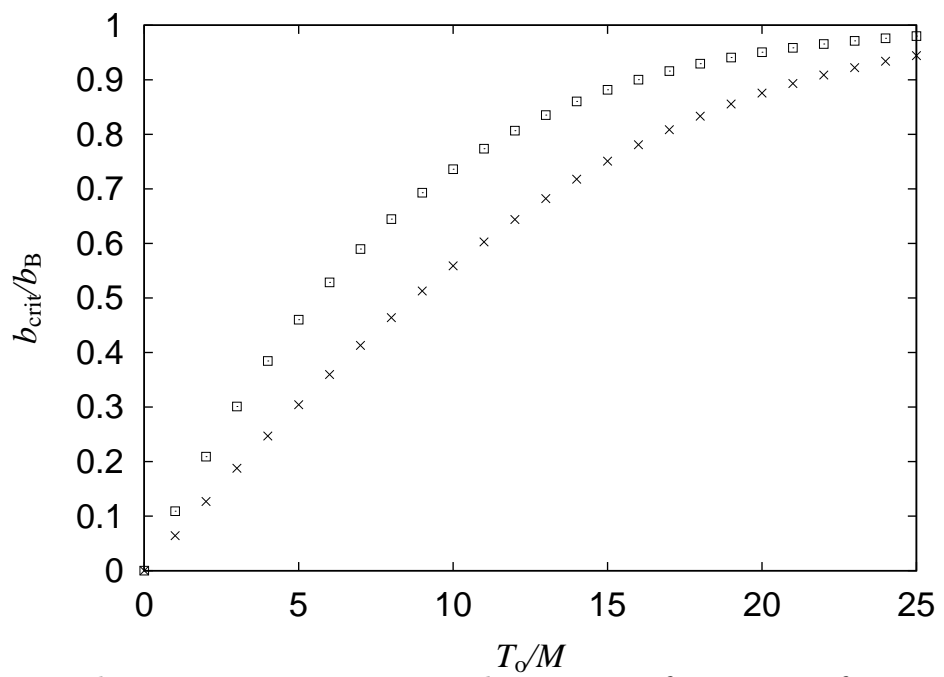

FIG. 6. We depict the impact parameter $b_{\text {crit }}$ as a function of time $T_{\mathrm{o}}$ at the observer $R_{\mathrm{o}}=100 \mathrm{M}$. The crosses are the data for the background self-similar dust chosen so that $W_{-}=\sqrt{2 / 10}$ while the squares correspond to the data of the background with $W_{-}=\sqrt{3 / 10}$. The impact parameter $b_{\text {crit }}$ grows monotonically and approaches to the value $b_{\mathrm{B}}=3 \sqrt{3} M$.

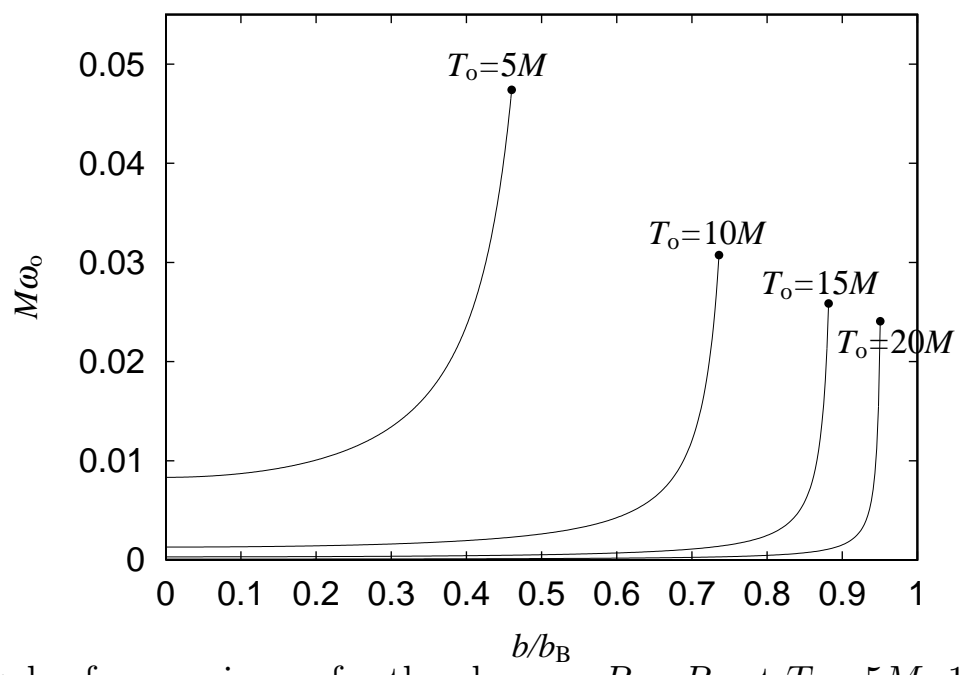

FIG. 7. The angular frequencies $\omega_{\mathrm{o}}$ for the observer $R=R_{\mathrm{o}}$ at $T=5 M, 10 M, 15 M$ and $20 M$ are depicted as a function of the impact parameter $b$. The null geodesics are assumed to start at $R=10^{-2} M$ with the comoving angular frequency $\omega_{L}=10^{2} M^{-1}$. The background self-similar dust is chosen so that $W_{-}=\sqrt{3 / 10}$. The angular frequency $\omega_{\mathrm{o}}$ is a monotonically increasing function of $b$. 


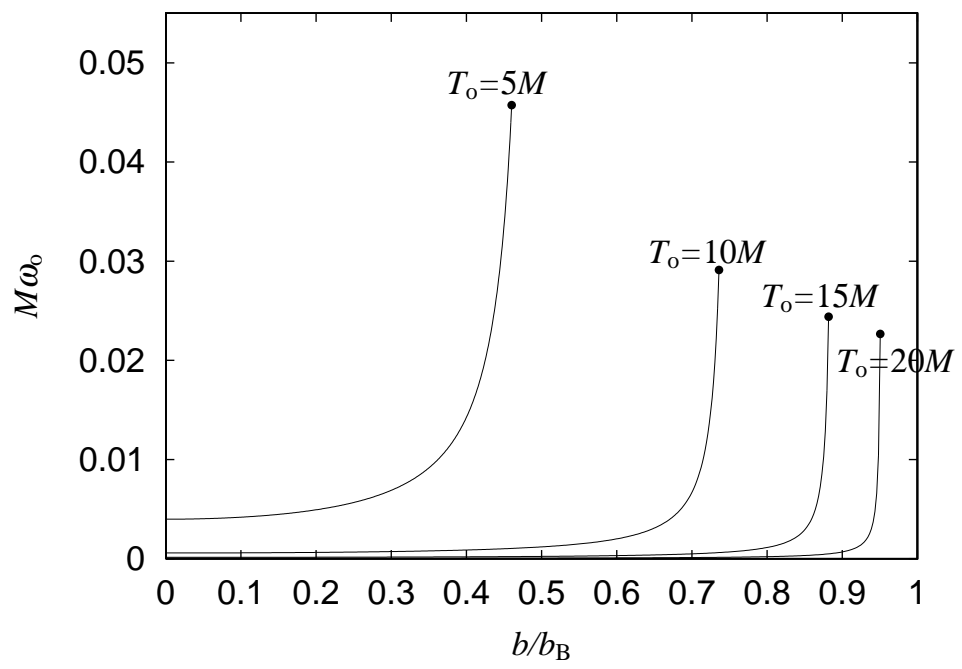

FIG. 8. The same as Fig.7 but the null geodesics are assumed to start at $R=10^{-3} M$ with the comoving angular frequency $\omega_{L}=10^{3} M^{-1}$.

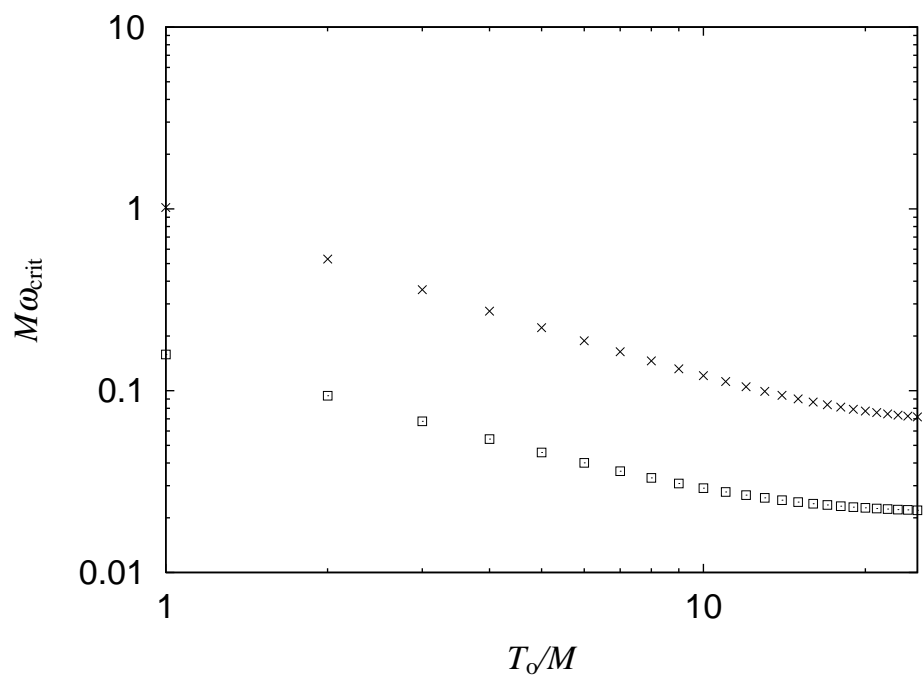

FIG. 9. We depict the observed angular frequency $\omega_{\text {crit }}$ of the null geodesic with the largest impact parameter $b=b_{\text {crit }}$ as a function of time $T_{\mathrm{o}}$ at $R_{\mathrm{O}}=100 \mathrm{M}$, where $\omega_{L}$ is set to be $10^{3} M^{-1}$. The crosses are the data for the the background self-similar dust chosen so that $W_{-}=\sqrt{2 / 10}$, while the squares correspond to the data of the background with $W_{-}=\sqrt{3 / 10}$. The observed angular frequency $\omega_{\text {crit }}$ approaches to some finite value for $T \rightarrow \infty$. 